\title{
Insider trading y el rol del gobierno corporativo
}

\author{
Carlos Orlando Rico Bonilla ${ }^{1}$
}

Recibido: 14 de abril de 2014

Aprobado: 20 de mayo de 2014

\section{Rico, C. (2014) Insider Trading y el rol del gobierno corporativo.}

\section{Activos 22, 25-37}

\section{Clasificación JEL: M14}

\section{Resumen}

En el presente documento se exponen algunos planteamientos de la literatura reciente sobre insider trading y los mecanismos de gobierno corporativo para prevenirlo y disminuirlo en el interior de la cultura organizacional. Se revisan los elementos más recientes de los conceptos sobre el tema y se plantean sus limitaciones en el contexto normativo y económico actual del país. También se indica cómo las propuestas de corporate governance 0 gobierno corporativo constituyen una opción a los problemas planteados por el insider trading.

1 Contador público, Universidad Nacional de Colombia. Docente de la Facultad de Ciencias Económicas. Correo electrónico: coricob@unal.edu.co 


\title{
Palabras clave
}

Información privilegiada, insider trading, gobierno corporativo, corrupción, asimetrías de información.

Rico, C. (2014). Insider trading and the role of corporate governance. Activos 22, 25-38

\begin{abstract}
This document describes some approaches from recent literature to insider training and corporate governance mechanisms to prevent and decrease it inside the organizational culture. The article reviews the most recent concepts on the subject and describes some of its limitations in the current regulatory and economic context of the country. It also indicates how the proposals for corporate governance represent a solution to the problems raised by insider trading.
\end{abstract}

\section{Keywords}

Privileged information, insider trading, corporate governance, corruption, information asymmetries.

Rico, C. (2014). Insider Trading et le rôle du management corporatife. Activos 22, 25-38

\section{Résumé}

Dans cet article, certaines approches de la littérature récente sur insider trading et les mécanismes de management corporatif de prévention et de diminution dedans la culture organisationnelle, sont exposés. Les éléments plus actuels des concepts sur le sujet sont examinés et ses limites dans le 
contexte réglementaire et économique actuel du pays sont soulevés. Il s'indique également comment les propositions pour corporate governance ou de management corporatif sont une option pour les problèmes soulevés par insider trading.

\section{Mots clés}

Opérations d'initiés, Insider Trading, management corporatif, la corruption, les asymétries d'information. 


\section{Introducción}

El usufructo de información privilegiada o insider trading es uno de los fenómenos más usuales de la incompatibilidad de los intereses y las expectativas corporativas, derivado de la separación entre propiedad y gestión empresarial. Además, es uno de los mayores riesgos que afrontan los inversionistas de cara a la conservación y protección de sus derechos de representación y beneficio en una participación patrimonial (Del Brío, 2006).

De hecho, como lo ejemplifican los escándalos financieros más relevantes de las últimas décadas (Parmalat, WorldCom, Lehman Brothers, entre otros), la base de los delitos de fraude empresarial incluía la explotación de asimetrías de información en favor de agentes internos (gerentes y consejeros) que asumieron conductas corruptas en detrimento de los actores externos que contaban con datos muy limitados (Kedia \& Philippon, 2005).

En el caso paradigmático de la célebre energética estadounidense Enron Corporation, las autoridades e investigaciones independientes demostraron con bastante rigurosidad cómo los más altos $\mathrm{CEO}$ aprovecharon su posición y conocimiento de monopolio de la situación financiera de la compañía para enriquecerse a costa de los proveedores, los empleados, los acreedores, los accionistas, el gobierno y más extensamente la sociedad en general.

No obstante su importancia, es aún un tema muy poco estudiado en el marco de la investigación financiera y contable. A pesar de los escándalos y crisis, no ha sido fácil establecer una legislación comercial y penal sobre la materia en general, o particular, en los mercados bursátiles (Maug, 2000; Del Brío, 2006).

Es de advertir que el objetivo central de este documento es señalar que contra la figura del insider trading la respuesta más óptima es el gobierno corporativo. En este sentido, el presente documento se divide en dos apartados: en el primero se revisa con un poco más de detalle el tema del insider trading, mientras que en la segunda se referencian las buenas prácticas de 
gobierno como un instrumento básico para reducir esta problemática, y por esta vía garantizar la protección de los inversionistas y demás stakeholders. Finalmente, se expone la conclusión.

\section{Insider trading}

Se describen a continuación tres aspectos encontrados en la bibliografía seleccionada:

\section{Delimitación técnica y jurídica}

Es bastante complejo determinar cuándo el uso que se hace de información privada por parte de los gestores o un grupo de accionistas de una organización implica un privilegio del que se derivan rendimientos arbitrarios. Como se señaló anteriormente, solo cuando se exhiben los hechos fraudulentos o ventajosos, se reconoce la figura del insider trading. Entonces, una de las primeras dificultades que se ha encontrado en la literatura es la delimitación técnica en sí misma de esta situación.

Hasta épocas muy recientes, el tema se ha circunscrito a la compra $\mathrm{y}$ venta de acciones o bonos que hacen los gerentes o consejeros de sus empresas administradas o aconsejadas (insider trading bursátil). ¿Cómo las motivaciones y la frecuencia de dichas transacciones pueden llegar a implicar la voluntad de querer afectar el precio en la Bolsa de un título en provecho de un objetivo determinado?

Si un chief executive officer (CEO) vende una porción de la participación patrimonial que recibe de la compañía como un beneficio adicional al salario, ¿se debe entender que tiene, por ejemplo, un interés en ajustar los indicadores de la empresa en términos de liquidez, carga impositiva o sostenibilidad? Si un grupo de la alta dirección compra valores, ¿ es probable que esta sea una señal de que busca generar una expectativa positiva sobre el futuro de la empresa, que tal vez no es real? 
Es claro que esto no necesariamente aplica de esta manera; entonces, identificar y tipificar estas conductas es un reto para la política y regulación de los países y, en particular, del gobierno corporativo de cada sociedad (Betzer \& Theissen, 2007).

Es así que en el estudio de Bhattacharya \& Daouk (2002) se examina, desde la década de los noventa, la legislación subyacente sobre protección del inversionista de 103 países que tienen mercados de valores activos. Se encontró que tan solo en 87 naciones hay reglas que se pueden asociar a la mitigación de prácticas de insider trading y que al evaluarlas a penas en 38 hay una efectividad relativa de su cumplimiento.

Tanto la reglamentación nacional como el gobierno corporativo corresponden a las tradiciones de derecho locales, la cultura de negocios y la institucionalidad económica. Entonces, hay diferentes posturas respecto de castigar o no, regular o no ciertos comportamientos que perfectamente se podrían asociar con insider trading (Betzer \& Theissen, 2007).

\section{Relación con la manipulación o el ajuste de la información financiera}

Tener una información privilegiada puede generar incentivos para manipular o bajar la calidad del reporte de las cifras financieras públicas, es un evento de insider trading corporativo. La gerencia usa su posición y conocimiento para usurpar los derechos de agentes diferentes de los inversores (Maug, 2000; Clacher, Hillier \& Lhaopadchan, 2009).

En la investigación de Beneish y Vargus (2002) se encuentra evidencia empírica obtenida del análisis de indicadores financieros, según la cual las prácticas de insider trading están asociadas a la calidad de los estados financieros y sus revelaciones, cuando no con la formación misma del proceso contable y el uso de ajustes contables, como provisiones, valorizaciones y otros ajustes por causación. 
De igual forma, Chen \& Lo (2006) aseguran que la divulgación de información voluntaria es más restrictiva y conservadora cuando se presenta un insider trading activo, ya que esta puede generar inquietud sobre algún aspecto opaco o inconsistente. Si hay riesgos de litigio bajos por revelaciones complementarias, es aún poco probable que se produzcan reportes complementarios.

\section{Insider trading en Latinoamérica}

Es muy poco lo que se ha revisado del asunto en el contexto latinoamericano. Un documento del Banco Interamericano de Desarrollo (BID) intenta medir la probabilidad de que se presente insider trading en siete países de la región (Argentina, Chile, México, Brasil, Colombia, Perú y Venezuela), de acuerdo con un conjunto de variables asociadas a la protección de los inversores, la economía del país, algunos sectores y una muestra de datos contables y bursátiles de 288 compañías. Por ejemplo, una de las preguntas base es la siguiente: ¿hay riesgos de expropiación de los activos?

El estudio indica que la estructura legal y económica de nuestros países podría abrir la puerta al insider trading por fenómenos característicos, como el secretismo empresarial, la concentración de la propiedad, las sociedades poco abiertas, el escaso nivel de financiación vía mercado de valores, la baja penetración de los servicios financieros, entre otros.

El texto enfatiza igualmente en que uno de los factores que más induce al aumento de la probabilidad de presentación de insider trading es la alta impunidad que se asocia a los fraudes financieros. Los procesos judiciales por delitos de cuello blanco o agentes económicos corruptos son demorados y de pocos resultados. Esto se percibe como una inseguridad para los inversionistas, particularmente los minoritarios, y genera, por ende, costos de capital más altos (Cruces \& Kawamura, 2005).

Finalmente, es de resaltar que esta investigación llama la atención sobre los avances del gobierno corporativo como un instrumento fundamental en 
la lucha contra el insider trading, más adecuado incluso que las regulaciones y leyes nacionales. Esta es precisamente la argumentación subyacente del siguiente epígrafe.

\section{El rol del gobierno corporativo y la protección del inversionista}

El asunto central del gobierno corporativo es cómo desarrollar y mantener un sistema de relaciones empresariales estables a largo plazo en presencia de comportamientos oportunistas de los diferentes stakeholders (Fernández y Gómez, 1999). Es decir, cómo limitar los diferentes conflictos de agencia, entre ellos el insider trading, un problema más exactamente de poder y asimetría de la información. La pregunta es recurrente: ¿cómo, por intermedio del triángulo de agentes (gestores, consejo de administración, propietarios) se alinean las diversas expectativas para fortalecer el bien general de la organización? Hay varios aspectos clave en las buenas prácticas sobre la materia, entre los que se destacan los siguientes:

\section{Los sistemas de remuneración y nombramiento de directivos}

El primer elemento relacionado con el control del insider trading es el que permite responder a la pregunta ¿cuál es el nivel óptimo de remuneración de los directivos, que les genere un incentivo para no involucrarse en esta práctica? Este es, sin lugar a dudas, un aspecto central y difícil.

En contextos donde se cumplen con más intensidad las leyes y sanciones existentes en contra del insider trading, se tiende a remunerar a los altos directivos con instrumentos de patrimonio de la empresa, un mecanismo de más pertinencia si se quiere motivar el compromiso y la responsabilidad de los gerentes con la firma (Denis \& Xu, 2013).

Cuando, por el contrario, se presentan entornos con prácticas de insider trading más expansivas, los beneficios e incentivos se materializan en pagos 
adicionales al salario (Denis \& Xu, 2013). Esta es, sin duda, una afirmación interesante, pues se tiende a pensar que precisamente con el ánimo de subir el valor de sus acciones, los altos directivos recurren al insider trading, pero quizá este no sea el único objetivo.

En este sentido, hoy en día es claro que el liderazgo y la ética corporativa son elementos constitutivos de la calidad de los gobiernos corporativos. Por esto son fundamentales la estructura y la transparencia asociadas a las remuneraciones de los altos directivos. En la actualidad es contraproducente promover la constitución de equipos directivos cerrados y aislados de la organización, funcionarios que se dedican a distribuir actividades o, como se dice coloquialmente, «simplemente a mandar».

Hay dos mecanismos muy importantes de buenas prácticas que quizá todavía no despegan con fuerza en nuestros países latinoamericanos: la formación y la autoridad de los comités de nombramientos y remuneraciones. De aplicarse adecuadamente estos dos mecanismos internos, pueden llegar a transmitir con firmeza la decisión de las empresas por incorporar parámetros modernos de participación de diferentes stakeholders y responsabilidad social corporativa, esto sin contar con los estímulos de competitividad que promueven entre todos los empleados de las compañías.

En este tema en particular hay que añadir la necesidad de divulgación de información financiera y no financiera. La regulación IFRS exige bastantes revelaciones sobre estos aspectos: salario de la alta gerencia, pagos basados en acciones y beneficios a empleados. Entre menos datos se revelen, más probabilidad habrá de ejercer insider trading.

\section{Tamaño e independencia de los consejos de administración}

La escasa literatura sobre insider trading y gobierno corporativo se ha enfocado en estudiar cómo el tamaño del consejo de administración y la 
independencia de sus integrantes incide en la reducción del uso de información privilegiada.

Se ha encontrado evidencia bastante relativa de que consejos de administración muy grandes o demasiado pequeños influyen en la generación de prácticas de insider trading; en el primer caso, esto courre porque el control que se puede ejercer sobre la gerencia es difuso y menos exhaustivo; en el segundo caso, porque se producen incentivos para que los pocos consejeros se confabulen con los altos directivos en el usufructo de los datos privilegiados (Rozanov, 2008).

Mientras tanto, consejos de administración poco independientes tienden a favorecer el uso de insider trading como un factor de colaboración con la gerencia relacionada. Por otra parte, aunque una mayor proporción de consejeros independientes facilita la denuncia y los incentivos para restringir económica y éticamente estas prácticas, también se ha demostrado el aprovechamiento de estos rangos para usufructuar asimetrías de información en su beneficio particular (Ravina \& Sapienza, 2010).

En fin, a pesar de lo poco estudiado del tema, se entiende que el rol del consejo es muy importante, y una configuración adecuada de este no es un asunto menor en el marco de la constitución del gobierno corporativo de una empresa. El consejo restringe posibles excesos de discrecionalidad y autoritarismo del gerente de cara a los inversionistas. Es claro que cumple más que una función de vigilancia, cooperación y estrategia entre diferentes agentes.

Preocupa que en América Latina este sea uno de los aspectos en los que menos se ha avanzado, y que falta por mejorar y ajustar las políticas estatales y corporativas (Cruces y Kawamura, 2005).

\section{Comités de auditoría y auditorías externas}

Los auditores externos no son en sí mismos un mecanismo de gobierno corporativo, pero es necesario advertir que un buen direccionamiento de 
su elección y contrato por parte de los comités de auditoría sí puede llegar a facilitar la documentación de prácticas de insider trading en la organización, como fruto de su actividad de revisión y evaluación, especialmente en las auditorías de gestión (Chen, Martin \& Wang, 2013).

El problema viene cuando falta independencia en el trabajo de este auditor; lo que sucede es que se convierte en un guardián del insider trading. Qué mejor modelo que la firma internacional Arthur Andersen \& Co, auditora de la ya bastante mencionada Enron. La estrecha relación de los funcionarios de las entidades - puerta giratoria de empleados y consejeros, y de múltiples y suntuosos contratos de consultoría- llevó a que se sistematizara la explotación de la información privilegiada.

En lugar de disminuir las asimetrías y garantizar la veracidad de los datos financieros, se legitimó el insider trading como parte de la cultura y el gobierno corporativo. Por eso no es de extrañar que desde entonces, y con la expedición de la Ley SOX, se implementaran medidas para condicionar el vínculo contractual del auditor con sus clientes, como la rotación obligatoria, por ejemplo (Rico, 2004).

\section{Conclusiones}

La figura del insider trading tiene potencial como campo de investigación contable y financiera. Así mismo, es un fenómeno para restringir con fuerza, ya que, en últimas, es asimetría de información y conductas oportunistas y fraudulentas de la gestión de las empresas. Un gobierno corporativo riguroso se convierte en un mecanismo sólido para la prevención y censura de estas prácticas.

En este sentido, de acuerdo con lo planteado en este documento, se evidencia que la literatura entiende como mecanismos más acertados de combate al insider trading, instrumentos como una estructura clara de incentivos y remuneraciones a los altos directivos, consejos de administración independientes y bien construidos, auditorías de gestión rigurosas, la 
emisión de una ley o reglamentación general expedida por una autoridad gubernamental, todos ellos instrumentos que no dejan de ser importantes.

En Latinoamérica, estos temas están aún en una etapa de relativo e incipiente desarrollo, que en particular se deben complementar con un esquema de cumplimiento efectivo y respeto por los derechos y la protección de los inversionistas.

\section{Referencias bibliográficas}

Beneish, M., \& Vargus, M. (2002). Insider trading, earnings quality and accrual mispricing. Accounting Review, 77(4), 755-791.

Betzer, A., \& Theissen, E. (2007). Insider trading and corporate governance: the case of Germany. Working Paper, University of Cologne.

Bhattacharya, U., \& Daouk, H. (2002). The world price of Insider trading. The Journal of Finance, 57(1), 75-108.

Chen, C., Martin, X., \& Wang, X. (2013). Insider trading, litigation concerns, and auditor going-concern opinions. Accounting Review, 88(2), 365-393.

Chen, Q., \& Lo, K. (2006). Insider trading and voluntary disclosures. Journal of Accounting Research, 44(5), 815-848.

Clacher, I., Hillier, D., \& Lhaopadchan, S. (2009). Corporate insider trading: a literature review. Revista Española de Financiación y Contabilidad, 143, 373-397.

Cruces, J., \& Kawamura, E. (2005). Insider trading and corporate governance in Latin America. Working Paper. Inter-American Development Bank.

Del Brío, E. B. (2006). Medición del insider trading en el mercado de valores español. Revista Española de Financiación y Contabilidad, 128, 115-136.

Denis, D., \& Xu, J. (2013). Insider trading restrictions and top executive compensation. The Journal of Accounting and Economics, 56, 91-112.

Fernández, A. y Gómez, S. (1999). El gobierno de la empresa: mecanismos alienadores y supervisores de las actuaciones directivas. Revista Española de Financiación y Contabilidad, 100, 355-380.

Kedia, S., \& Philippon, T. (2005). The economics of fraudulent accounting. Working Paper. National Bureau of Economics Research. 
Maug, E. (2000). Insider trading legislation and corporate governance. Working Paper. Duke University.

Ravina, E., \& Sapienza, P. (2010). What do independent directors know? Evidence from their trading. Review of Financial Studies, 23, 962-1003.

Rico, C. (2004). Globalización, audit expectation gap y rotación obligatoria de auditores. Revista Internacional Legis de Contabilidad y Auditoría, 24, 8-47.

Rozanov, K. (2008). Corporate governance and insider trading. Working Paper. London Business School. 